\title{
Size-Selected Epitaxial Nanoislands Underneath Graphene Moiré on $\operatorname{Rh}(111)$
}

\author{
Muriel Sicot, ${ }^{\dagger, \S, *}$ Philipp Leicht, ${ }^{+, \S}$ Andreas Zusan, ${ }^{\dagger}$ Samuel Bouvron, ${ }^{\dagger}$ Ole Zander, ${ }^{\dagger}$ Martin Weser, ${ }^{\dagger}$ \\ Yuriy S. Dedkov, ${ }^{\ddagger, \perp}$ Karsten Horn, ${ }^{\ddagger}$ and Mikhail Fonin ${ }^{\dagger, *}$ \\ ${ }^{\dagger}$ Fachbereich Physik, Universität Konstanz, 78457 Konstanz, Germany and ${ }^{\ddagger}$ Fritz-Haber-Institut der Max-Planck-Gesellschaft, 14195 Berlin, Germany. ${ }^{\circledR}$ These authors \\ contributed equally to this work. ${ }^{\perp}$ Present address: SPECS Surface Nano Analysis GmbH, Voltastrasse 5, 13355 Berlin, Germany.
}

$\mathrm{T}$ he investigation of interfaces between graphene ${ }^{1,2}$ and metals is of great technological importance because of recently demonstrated large-scale productions of high-quality graphene on metal substrates. ${ }^{3,4}$ Furthermore, graphene/ metal interfaces are of crucial importance for the preparation of conventional ${ }^{5}$ or spinpolarized $^{6,7}$ contacts in devices and attract considerable interest due to the possibility to fabricate graphene-based spintronic units. $^{8,9}$ In general, the properties of epitaxial graphene/substrate interfaces can be efficiently controlled by inserting other materials between graphene and the original support, a process referred to as intercalation. ${ }^{10-13}$ In the particular case of graphene grown epitaxially on transition metals, intercalation of layers of alkali metals, noble metals, or oxygen has already been successfully used to tune the hybridization between the electronic states of graphene and the underlying substrate in order to restore the electronic structure of freestanding graphene or to induce doping. ${ }^{11,12,14-17}$

Focusing on the graphene/ferromagnet systems, the use of intercalation as an alternative to the direct epitaxial growth of graphene on ferromagnetic surfaces, for example, $\mathrm{Ni}(111)^{12,18}$ and $\mathrm{Co}(0001),{ }^{19,20}$ may offer several advantages in an applications perspective. First, the intercalated materials can be stabilized in structures highly unfavorable for their bulk counterparts. ${ }^{21}$ Second, interfaces composed of hexagonally close-packed ferromagnetic surfaces such as $\mathrm{Ni}(111)^{18}$ and $\mathrm{Co}(0001)^{19}$ in contact with graphene are considered to play an important role in the field of graphenebased spintronics as they are predicted to exhibit large spin filtering efficiencies. ${ }^{8,9}$ The intercalation approach could hereby serve as a flexible method to produce perfect

ABSTRACT We use in situ scanning tunneling microscopy (STM) to investigate intercalation of the ferromagnetic $3 \mathrm{~d}$ metals $\mathrm{Ni}$ and $\mathrm{Fe}$ underneath a graphene monolayer on $\mathrm{Rh}(111)$. Upon thermal annealing of graphene/Rh(111) with the deposited metal on top, we observe the formation of epitaxial monatomic nanoislands grown pseudomorphically on $\mathrm{Rh}(111)$ and covered by graphene. The size and shape of intercalated nanoislands is strongly influenced

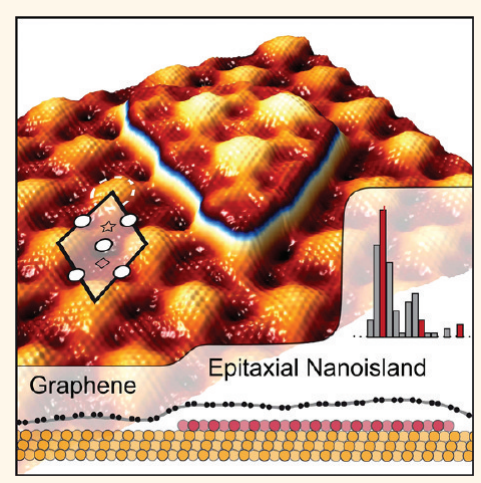
by the local spatial variation of the graphene-Rh bonding strength. In particular, the side length of the intercalated nanoislands shows maxima around discrete values imposed by the periodicity of the graphene moiré. Intercalation can be performed efficiently and without any visible damage of the graphene overlayer in the studied temperature range between 670 and $870 \mathrm{~K}$. We identify the main intercalation path to be via diffusion through pre-existing lattice defects in graphene, accompanied by the second mechanism which is based on the material diffusion via metal-generated defects followed by the defect healing of the graphene lattice. We deem these graphene-capped and sharply confined ferromagnetic nanoislands interesting in the fields of spintronics and nanomagnetism.

KEYWORDS: graphene $\cdot$ moiré $\cdot$ rhodium $\cdot$ ferromagnet $\cdot$ intercalation . scanning tunneling microscopy · photoemission spectroscopy

epitaxial graphene/ferromagnet systems, which are needed for the future spintronic applications. Third, intercalation of ferromagnets in conjunction with periodically corrugated graphene on lattice-mismatched surfaces of $\mathrm{Pt}(111),{ }^{22-24} \operatorname{Ir}(111),{ }^{23,25-27} \mathrm{Rh}$ $(111),{ }^{23,28-32}$ and $\mathrm{Ru}(0001)^{23,28,33-35}$ would possibly allow the preparation of arrays of magnetic nanostructures covered by a passivating graphene layer ensuring chemical stability of the system toward oxidation in air. ${ }^{36}$ Although several studies on the intercalation of ferromagnetic materials between graphene and supporting metal have been reported, ${ }^{21,37-39}$ little is known about the effects of the lateral variation in binding strength

\begin{abstract}
* Address correspondence to sicot_muriel@yahoo.fr, mikhail.fonin@uni-konstanz.de.
\end{abstract}

Received for review August 18, 2011 and accepted December 17, 2011.

Published online January 03, 2012 10.1021/nn203169j

C 2012 American Chemical Society 

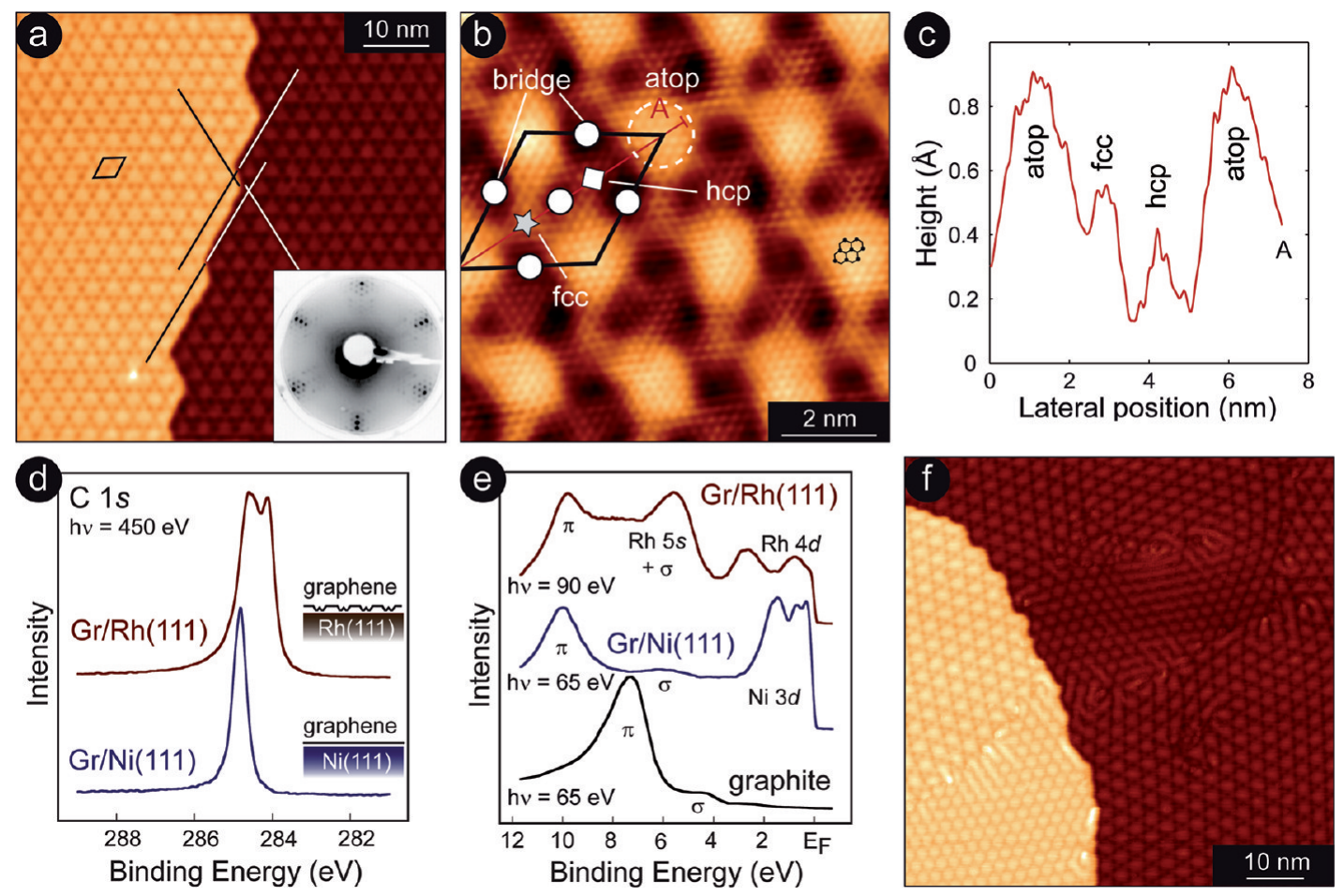

Figure 1. (a) STM topograph $\left(65 \times 65 \mathrm{~nm}^{2}\right)$ of the regular moiré pattern of graphene on $\operatorname{Rh}(111)\left(U_{\mathrm{T}}=1.1 \mathrm{~V} ; I_{\mathrm{T}}=0.18 \mathrm{nA}\right)$. The inset in (a) shows the corresponding LEED image $\left(E_{\mathrm{e}}=58 \mathrm{eV}\right)$. (b) Atomically resolved $9 \times 9 \mathrm{~nm}^{2}$ STM image of the moiré structure $\left(U_{T}=0.47 \mathrm{~V} ; I_{T}=4.2 \mathrm{nA}\right)$. The black rhombus outlines the moiré supercell. (c) Height profile along the long diagonal of the moiré supercell. (d) C $1 \mathrm{~s}$ core-level photoemission spectra of the graphene layer on $\mathrm{Rh}(111)$ and of the graphene layer on $\mathrm{Ni}(111)(h v=450 \mathrm{eV})$. (e) Valence-band photoelectron spectra of graphene/Rh(111) $(h v=90 \mathrm{eV})$, graphene/Ni(111) $(h v=65$ $\mathrm{eV}$ ), and graphite single crystal ( $h v=65 \mathrm{eV}$ ) acquired in the normal emission geometry. (f) STM topograph $\left(75 \times 75 \mathrm{~nm}^{2}\right)$ of a graphene layer with structural defects due to the presence of different moiré domains $\left(U_{\mathrm{T}}=0.14 \mathrm{~V} ; I_{\mathrm{T}}=0.42 \mathrm{nA}\right)$.

due to the moiré structure on the morphology of intercalated material.

In this work, we report on the intercalation of the ferromagnetic 3d metals $\mathrm{Ni}$ and Fe underneath graphene on Rh(111). We show that the shape and size of intercalated metal islands is strongly influenced by the intricate spatial binding strength variation at the graphene/Rh(111) interface. On the basis of our scanning tunneling microscopy (STM) results at different stages of the intercalation process, possible intercalation mechanisms are discussed.

\section{RESULTS AND DISCUSSION}

Figure 1a represents a large-scale topographic STM image showing the moiré structure of graphene/Rh(111) exhibiting a high crystalline quality. A large graphene domain continuously spreads across several substrate steps without visible defects on the atomic scale. Substrate steps are aligned to the main crystallographic directions of the graphene layer as a result of substantial reshaping of the $\mathrm{Rh}(111)$ substrate during the preparation. A moiré periodicity of $2.9 \pm 0.05 \mathrm{~nm}$ is found from low-energy electron diffraction (LEED) investigations (inset in Figure 1a) corresponding to approximately 12 times the lattice constant of graphene and 11 times that of $\mathrm{Rh}(111)$. This is in good agreement with experimental values reported earlier ${ }^{32}$ as well as with the theoretical moiré periodicity of $d_{\mathrm{m}}=$ $2.88 \mathrm{~nm}$ derived as described in ref 26 with $a_{\mathrm{C}}=2.46 \AA$ and $a_{\mathrm{Rh}}=2.69 \AA$. Figure $1 \mathrm{~b}$ shows an atomically resolved STM image of graphene/Rh(111). Main features of the moire cell of graphene/Rh(111) are bright areas marked by dashed circles and deep depressions, one in the middle of the moiré cell and four at the moiré cell border, each of them marked with filled circles. On STM images, three of these depressions constitute a triangle. Hence, the point enclosed by the triangle of depressions (marked by a filled square) is not equivalent to the mirror-symmetric position on the other half of the moiré cell (marked by a filled star). More precisely, the area depicted by the filled square in the center of the triangle is darker compared to the equivalent site marked by a filled star. This situation becomes more obvious in a line profile along the long diagonal of the moiré unit cell in Figure 1c. Assuming a commensurate 12 carbon/11 Rh supercell and a perfect alignment of graphene and rhodium lattices, we can deduce the local configuration of the carbon atoms in the graphene layer with respect to the substrate. In Figure $1 b$, the configurations are assigned to the apparent STM contrast: bright areas mark regions, where the center of the carbon ring is placed above a substrate atom, further on referred to as atop positions. The appearance of the bright atop areas 

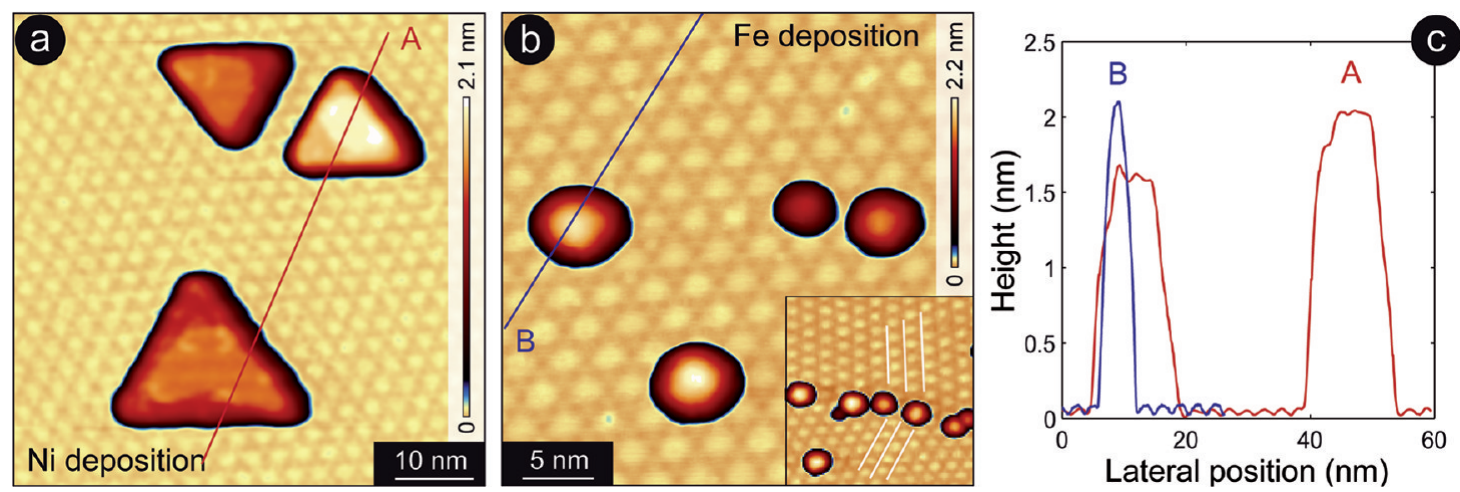

Figure 2. (a) STM topograph $\left(60 \times 60 \mathrm{~nm}^{2}\right)$ of predeposited $\mathrm{Ni}$ on graphene/Rh(111) $\left(U_{\mathrm{T}}=1.54 \mathrm{~V} ; l_{\mathrm{T}}=1.14 \mathrm{nA}\right)$. (b) STM topograph $\left(33 \times 33 \mathrm{~nm}^{2}\right)$ of predeposited Fe on graphene/Rh(111) $\left(U_{T}=1.11 \mathrm{~V} ; I_{T}=0.49 \mathrm{nA}\right)$. Inset in (b) shows a $34 \times 34 \mathrm{~nm}^{2}$ STM image illustrating preferential nucleation of Fe clusters at a domain boundary. The main directions of two moiré patterns are outlined in both domains by white lines $\left(U_{T}=1.29 \mathrm{~V} ; I_{T}=0.60 \mathrm{nA}\right)$. (c) Height profiles measured along the lines in (a) and (b), respectively.

coincides with the findings on graphene//r $(111)^{25,26}$ and graphene/Ru(0001) $)^{33}$ systems. The interconnecting regions between the dark depressions can be identified referring to the position of the carbon ring center located either above three-fold hollow hcp (marked by a solid square) or fcc (marked by a solid star) substrate sites, respectively. The assignment of $\mathrm{fcc} / \mathrm{hcp}$ areas within the moiré cell was performed based on comparison of the STM apparent height values with the density functional theory (DFT) calculations in ref 31. The dark depressions in our STM images coincide with bridge sites, where carbon rings are located above bridging substrate positions. Indeed, theoretical calculations predict bridge sites to be energetically favorable for graphene on $\mathrm{Ru}(0001)^{35}$ as well as on $\mathrm{Rh}(111) .^{31}$

The spatial modulation of the apparent vertical height of the graphene sheet in STM depends on the scanning parameters and varies between 0.5 and $1.5 \AA$. The large corrugation in STM images gives a strong indication for a lateral variation in bonding strength due to two contributing factors: first, a variation in bonding strength leads to a physical corrugation of the graphene sheet; ${ }^{35,40}$ and second, the laterally inhomogeneous accumulation of charge carriers modifies the density of states and work function locally. ${ }^{31}$ A separation of both effects is not unambiguously possible by STM; however, the comparison with DFT data ${ }^{31}$ suggests a correlation of the STM apparent height with the physical corrugation of the graphene sheet.

Core-level and valence-band photoemission spectroscopy (PES) provides a strong support for the considerable local variations in bonding strength between the carbon layer and the $\mathrm{Rh}(111)$ substrate. In Figure 1d,e, PES measurements of corrugated graphene on $\mathrm{Rh}(111)$ and flat graphene on $\mathrm{Ni}(111)$ are compared. For graphene/Rh(111), the $C$ 1s peak is split in two distinct components with an energy separation of about $0.44 \mathrm{eV}$ (see Figure 1d), whereas only one single $C$ is line is present for graphene/Ni(111), at a position which cor- responds to the binding energy of the more strongly bound component of graphene/Rh(111). In the case of graphene/Rh(111), the splitting of the $\mathrm{C} 1 \mathrm{~s}$ line in two components is contributed to the coexistence of regions with differing bonding strength. ${ }^{23}$ Recent DFT calculations show that the double peak structure of the C 1s line is formed by a superposition of single lines with a continuous rather than binary distribution of binding energies arising from a locally different degree of interaction between graphene and the underlying metal. Hereby the distribution of lines shows two intensity maxima forming the experimentally observed C 1 s double peak. ${ }^{41}$ In Figure 1e, the valenceband PES spectra acquired in normal emission are shown. Compared to graphite, the $\pi$-bands of graphene/Ni(111) and graphene/Rh(111) show a large shift to higher binding energies, indicating a strong overall bonding to the substrate. The difference in binding energy of the $\pi$-states in graphene/ $\mathrm{Rh}(111)$ and pure graphite amounts to about $2.3 \mathrm{eV}$, which is close to the value for graphene/Ni(111) ${ }^{18}$ and also for graphene/Ru(0001), ${ }^{42}$ indicating a comparable bonding strength of the graphene layer on top of the $\mathrm{Rh}(111)$ surface. However, a considerably different local redistribution of the bonding strength in graphene/Rh(111) compared to $\mathrm{Ru}(0001)$ or $\operatorname{Ir}(111)$ can be deduced from strong contrast variations observed by STM.

In the following, we will show that local bonding strength variation between graphene and metal support has a strong impact on the structure of $3 \mathrm{~d}$ metal islands intercalated underneath graphene on $\mathrm{Rh}(111)$. Furthermore, we find that lattice defects in the graphene layer, such as point defects and domain boundaries (see Figure 1f), play an important role in the intercalation process. Therefore, in the following, we will first briefly discuss the growth of the ferromagnetic metals on top of the graphene/Rh(111) system and subsequently focus on the morphology of the intercalated islands underneath graphene/ $\mathrm{Rh}(111)$ as well as on the discussion of possible intercalation routes. 

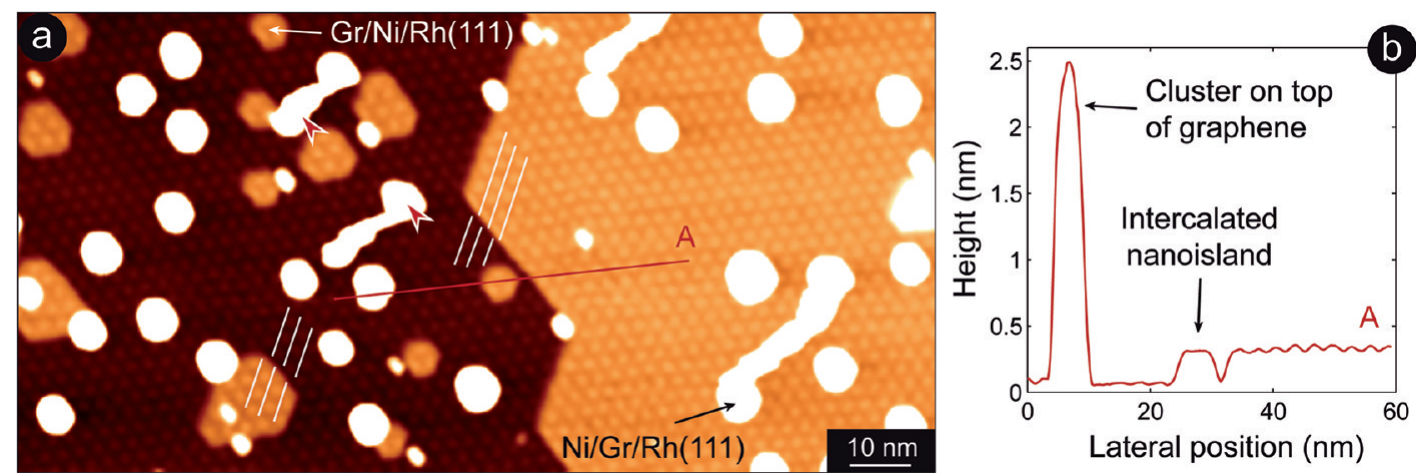

Figure 3. (a) Large-scale $150 \times 75 \mathrm{~nm}^{2}$ STM topograph obtained after Ni deposition followed by annealing up to $870 \mathrm{~K}$. The red arrows point at Ni clusters on top of graphene swept by the STM tip $\left(U_{T}=1.20 \mathrm{~V}, I_{T}=1 \mathrm{nA}\right)$. (b) Height profile along the red line in (a) highlighting the distinct difference between the $\mathrm{Ni}$ clusters on top of graphene and intercalated nanoislands.

Examples for either $\mathrm{Ni}$ or Fe intercalation will be discussed as these materials showed the same behavior during the experiments.

At the first step, $\mathrm{Ni}$ and Fe were predeposited on graphene/Rh(111) at room temperature. The nominal deposition of $2 \AA$ of $\mathrm{Ni}$ leads to the formation of large, flat, and triangular-shaped islands ${ }^{30}$ with the edges aligned approximately along the $\langle 1 \overline{1} 0\rangle$ directions of the $\mathrm{Rh}(111)$ substrate, as displayed in Figure 2a. Their average height is $1.8 \mathrm{~nm}$, and their size defined by the length of their longer edge is ranging from 5 to $18 \mathrm{~nm}$ (see the corresponding line profile in Figure $2 \mathrm{c}$ ). Figure $2 \mathrm{~b}$ represents a STM topograph of $1 \AA$ of nominal Fe deposited on graphene/Rh(111) at room temperature. The Fe clusters are of hemispherical shape with a histogram maximum at $9 \mathrm{~nm}$ in diameter. The typical height of the Fe clusters is $2 \mathrm{~nm}$, as shown in Figure 2c. Interestingly, graphene grain boundaries act as nucleation centers, as illustrated in the inset in Figure $2 b$, where many clusters nucleate at the boundary forming a cluster chain. This is in agreement with theoretical predictions showing that grain boundaries are more chemically reactive than the undisturbed graphene lattice ${ }^{43}$ as well as with experimental results reported for $\mathrm{Ni}$ on graphene/Ni(111), ${ }^{44}$ where an agglomeration of deposited material at defect-rich areas is observed, suggesting that defects in graphene act as nucleation centers. On the other hand, $\mathrm{Fe}, \mathrm{Ni}$, and $\mathrm{Co}$ are reported to reduce vacancy formation energies of graphene $^{45}$ and might also induce the formation of graphene defects.

The surface topography was found to change considerably after annealing of graphene/Rh(111) with predeposited $\mathrm{Ni}$ or Fe on top. Figure 3a shows a largescale STM topograph taken after $\mathrm{Ni}$ deposition on graphene/Rh(111) followed by a subsequent annealing up to $870 \mathrm{~K}$ for $20 \mathrm{~min}$. A single graphene layer exhibiting the regular moiré structure is observed. This graphene domain continuously covers two terraces, whereas the step edges are straight. The bending of the graphene layer at the step edge produces a lateral displacement of the moiré pattern, ${ }^{46}$ as outlined by two sets of white lines parallel to one of the $\langle 1 \overline{1} 0\rangle$ direction of the moiré structure, hence any bending/ lifting of graphene due to obstacles can be visually identified in STM by a lateral displacement of the moiré pattern across the obstacle. Two main features are seen in this image. First, compact Ni clusters appearing in white on the STM topograph can be observed. These clusters are sitting on top of graphene, deduced from the fact that they can be swept by the STM tip and consequently form elongated paths on the image marked by the red arrows. Second, flat hexagonalshaped islands exhibiting a moiré structure on their surface can be observed. The height of such islands coincides with the step height of graphene grown across substrate steps in contrast to the substantially higher clusters on top of graphene (white features in Figure 3a). This becomes clear from the height profile shown in Figure $3 \mathrm{~b}$. Upon repeated annealing, the number of clusters on top of graphene decreases and is accompanied by a growth of the area occupied by the flat islands. Moreover, annealing leads to a ripening of the clusters on top of graphene in conjunction with a substantial increase in diameter and height.

On the basis of the observation of graphene moiré on top of the hexagonal-shaped islands, we identify them as intercalated nanoislands (INIs) between the $\mathrm{Rh}$ (111) substrate and the covering graphene monolayer. The lateral displacement of the moiré pattern outlined in Figure $3 a$ by parallel white lines shows that the covering graphene layer is lifted seamlessly across the INI's edges. The formation of monolayer flat patches of $\mathrm{Ni}$ on top of graphene on the other hand can be excluded due to the existence of a graphene moiré pattern on top and the fact that annealing rather leads to the formation of round, nanometer high compact $\mathrm{Ni}$ clusters instead of hexagonal-shaped monolayer-thick islands. The same behavior was observed for Fe during all stages of the intercalation process (see also Figure 6 and corresponding text).

Now, we address in detail the morphology of the INIs. We found that the graphene moiré imposes registration on the compact INIs. The overall 

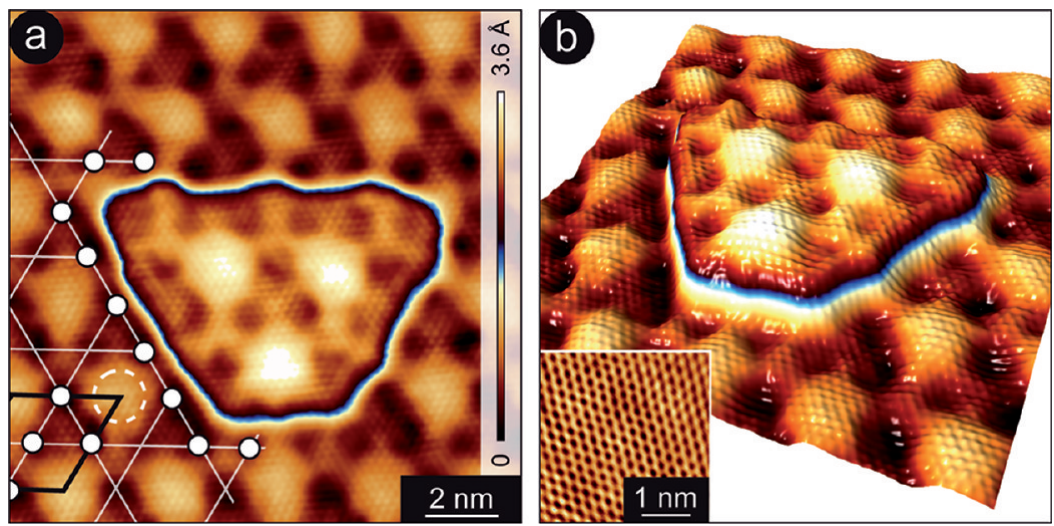

Figure 4. (a) Atomically resolved $14 \times 14 \mathrm{~nm}^{2}$ STM topograph obtained after room temperature deposition of Ni followed by annealing at $670 \mathrm{~K} \quad\left(\mathrm{U}_{T}=0.47 \mathrm{~V} ; I_{T}=4.2 \mathrm{nA}\right)$. White dots mark bridge sites of graphene/Rh(111). (b) Three-dimensional representation of the nanoisland in (a). Inset in (b) shows an atomically resolved STM topograph of graphene/Ni(111)/W(110) prepared as described elsewhere ${ }^{21,36}\left(3.5 \times 3.5 \mathrm{~nm}^{2} ; U_{\mathrm{T}}=0.002 \mathrm{~V} ; I_{\mathrm{T}}=42 \mathrm{nA}\right)$.
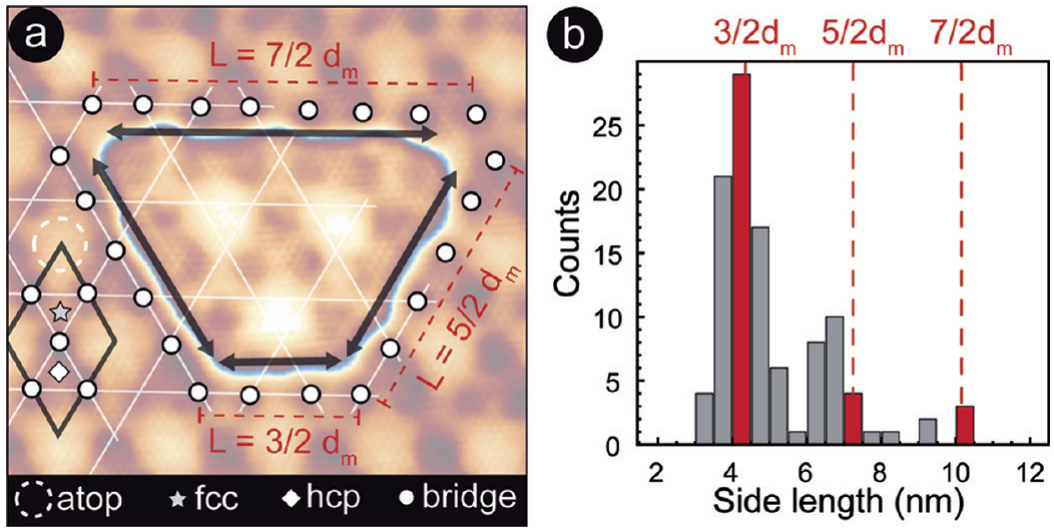

Figure 5. (a) Atomically resolved STM topograph of an intercalated Ni nanoisland. The shift of the moiré high-symmetry points upon intercalation of one additional pseudomorphically grown layer is illustrated. Red lines indicate the expected INI side length defined as the length of the bridge site row, whereas black arrows indicate the measured one. (b) Histogram with the distribution of the side lengths of intercalated $\mathrm{Ni}$ nanoislands compiled on the basis of 108 length measurements.

morphology of the INIs observed in Figure 3 differs strongly from the one of the $\mathrm{Ni}$ islands deposited either on top of graphene/Rh(111) or directly on the bare $\mathrm{Rh}(111)$ surface. ${ }^{47}$ The sides of the INIs are parallel to the $\langle 1 \overline{1} 0\rangle$ direction of the $\mathrm{Rh}(111)$ surface similarly to $\mathrm{Ni}$ growth at room temperature on $\mathrm{Rh}(111) .{ }^{47}$ However, in the latter case, the $\mathrm{Ni}$ nanoislands are of triangular shape due to diffusion along preferential directions. ${ }^{47}$ In the case of the INIs, the shape is rather hexagonal.

Atomically resolved STM topographs of graphenecovered Ni INIs allow one to extract further structural details. One typical Ni INI is displayed in Figure $4 a$,b. Graphene covering the surface of the Ni INIs exhibits an atomic arrangement identical to the one of graphene covering the neighboring $\mathrm{Rh}(111)$ surface as extracted from the analysis of the fast Fourier transform calculated from the STM topography on top of an INI and next to it (not shown here). As a result, the periodicities of the moiré on the graphene/Rh(111) and on the graphene/ $\mathrm{Ni} / \mathrm{Rh}(111)$ surfaces are found to be equal. Furthermore, upon intercalation, a shift of one-quarter of a moiré period between the moiré structure on graphene/Rh(111) and the moiré on top of the INI is observed (Figure 4a). This is explained by a permutation of high symmetry sites due to the intercalation of one monolayer $\mathrm{Ni}$ between graphene and $\mathrm{Rh}(111)$, as illustrated in Figure 5a. These results indicate that the intercalated material grows pseudomorphically on $\mathrm{Rh}(111)$, implying an adaption of the $\mathrm{Ni}$ interatomic distances to the $\mathrm{Rh}(111)$ lattice parameter.

The atomically resolved STM topograph in Figure 4a gives also a detailed view of the edges of INIs. The edge of the intercalated island follows strictly the rows formed by the dark spots of the moiré pattern, which are attributed to bridge sites. This feature was observed for all INIs and can explain the hexagonal shape of the INIs reflecting the symmetry of the moiré pattern. When the different sites of carbon rings within the moiré structure with respect to the underlying $\mathrm{Rh}(111)$ are recalled, the bridge sites are the regions where the $\mathrm{C}-\mathrm{Rh}$ interaction is strongest ${ }^{31}$ (see Figure $1 \mathrm{a}-\mathrm{e}$ ). Hence, they represent regions for which the graphene is strongly pinned to the Rh surface, thus 

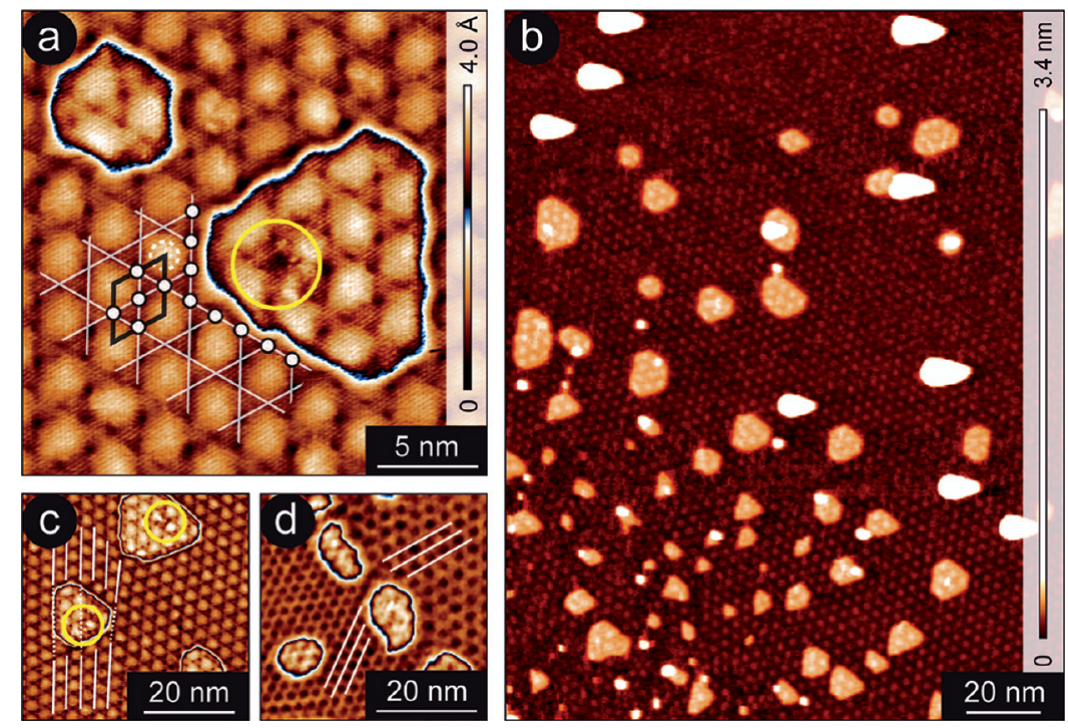

Figure 6. Fe intercalation at $870 \mathrm{~K}$. (a) Atomically resolved $23 \times 23 \mathrm{~nm}^{2} \mathrm{STM}$ topograph of two intercalated Fe nanoislands $\left(U_{T}\right.$ $\left.=0.02 \mathrm{~V} ; I_{T}=29.9 \mathrm{nA}\right)$. White filled circles mark the positions of the bridge sites. (b) STM topograph $\left(150 \times 190 \mathrm{~nm}^{2}\right)$ showing in the lower left corner a higher density of intercalated Fe nanoislands for disordered graphene. The elongated shape of the $\mathrm{Fe}$ clusters on top of graphene (white) is a scanning artifact $\left(U_{T}=1.2 \mathrm{~V} ; I_{T}=0.55 \mathrm{nA}\right)$. (c) STM topograph $\left(50 \times 50 \mathrm{~nm}^{2}\right)$ of graphene on $\mathrm{Rh}(111)$ demonstrating preferential intercalation at point defects $\left(U_{\mathrm{T}}=0.02 \mathrm{~V} ; I_{\mathrm{T}}=29.9 \mathrm{nA}\right)$. (d) STM topograph $(45 \times$ $\left.45 \mathrm{~nm}^{2}\right)$ in the dark atop contrast showing preferential intercalation of Fe at a graphene domain boundary $\left(U_{\mathrm{T}}=0.95 \mathrm{~V} ; I_{\mathrm{T}}=\right.$ $0.25 \mathrm{nA}$ ). Defects in the graphene layer are outlined in (a) and (c) by circles.

imposing considerable constraints on the material diffusion across the rows of bridge sites.

In Figure 5b, a histogram of the measured side lengths of INIs is shown. The distribution of side lengths yields maxima around discrete values of $(n+1 / 2) d_{m}$ with $n \in N$ and $d_{m}$ being the moiré period. The distance between two neighboring bridge sites corresponds to $1 / 2 d_{\mathrm{m}}$. The discrete side length of INIs can be explained by taking into account the intersection of lines connecting the bridge sites, which impose barriers for material diffusion underneath graphene. A closer look at the histogram in Figure $5 b$ reveals the maxima to be at slightly lower values compared to the series of lengths $3 / 2 d_{m}, 5 / 2 d_{m}$, and $7 / 2 d_{m}$. In Figure $5 a$, the expected side length defined by the bridge sites (red line) is compared to the measured one (black arrow). The observed small deviation arises obviously from a softening of the INI's corners due to partially unfilled areas within the boundaries of bridge sites.

In the following, possible intercalation routes are discussed upon the example of Fe. Figure 6 shows the result of Fe intercalation at $870 \mathrm{~K}$. A similar situation is observed here as found for the Ni INIs (see Figure 2b). The discrete side lengths of Fe INIs are particularly well illustrated in the STM topograph in Figure 6a. On single domain graphene terraces, one atomic layer thick Fe INIs with the same characteristics as previously discussed Ni INIs are observed, exhibiting the regular graphene moiré pattern on their surface. Most of the Fe INIs in Figure 6 exhibit a local perturbation of the moiré pattern [pointed out by circles in (a) and (c)], which we assign to defects in the graphene lattice.
These observations suggest that pre-existing lattice defects allow materials to efficiently penetrate the graphene overlayer and thus represent the most probable route for metal intercalation in this system. Figure $6 \mathrm{~b}$ displays a STM topograph taken after Fe intercalation on an inhomogeneous graphene/Rh(111) surface. High-quality graphene moiré is observed in the upper part of the image, whereas in the lower part, a large number of point defects and dislocations are visible. Only a small density of INIs is observed underneath good quality graphene, which are accompanied by several Ni 3D clusters on top of graphene. On the contrary, areas with a high defect density exhibit also a high density of INIs with only negligible amount of material remaining on top suggesting efficient intercalation. These results give clear evidence that penetration via pre-existing lattice defects is a very effective intercalation route. Examples for efficient intercalation through various defects are given in Figure $6 c, d$, respectively.

Nevertheless, the surface of some INIs does not exhibit any visible moiré perturbation, as illustrated by the small Fe INI shown in the top left corner of the STM topograph in Figure 6a. This is also observed in case of $\mathrm{Ni}$ intercalation (see the Ni INI in Figure 4). Here, alternative intercalation routes should be considered. One possible mechanism is intercalation via the formation of carbidic domains, which has recently been reported for $\mathrm{Ni}$ intercalation on graphene/Ni(111) at relatively low temperatures. ${ }^{44}$ Upon annealing of graphene/Ni(111) with the predeposited $\mathrm{Ni}$, the destruction of graphene and the formation of a Nicarbide surface phase was observed. Thereafter, 
carbidic domains allow $\mathrm{Ni}$ to intercalate, eventually reforming the perfect graphene layer on top at higher temperature after intercalation is complete. However, this large-scale intercalation route can be ruled out in our case since no areas with carbidic phases were observed. A further possible mechanism might be diffusion through metal-generated atomic-scale defects. Recent theoretical calculations ${ }^{45}$ show that transition metals ( $\mathrm{Fe}, \mathrm{Ni}$, and $\mathrm{Co}$ ) reduce vacancy formation energies of graphene and therefore might induce the formation of atomic-scale defects in graphene at elevated temperatures. Subsequently, metal atoms can diffuse through the induced defects and merge with the metal substrate underneath graphene. After the intercalation process is complete, the $\mathrm{C}-\mathrm{C}$ bonds are re-established, thus restoring the original graphene lattice. In our opinion, this is the second possible intercalation route aside from the diffusion via preexisting defects.

\section{CONCLUSION}

In conclusion, we studied the intercalation of Fe and Ni underneath epitaxial graphene grown on $\mathrm{Rh}(111)$ by means of scanning tunneling microscopy. We found that the intercalated material forms monatomic nanoislands grown pseudomorphically on $\mathrm{Rh}(111)$. Graphene on $\mathrm{Rh}(111)$ exhibits a moiré structure, which is distinctly different compared to those structures observed on other metal surfaces. The local spatial variation of the graphene-substrate bonding strength controls the size and shape of intercalated nanoislands. In particular, the side length of the INIs shows maxima around discrete values imposed by the periodicity of the graphene moiré, and INI shapes are predominantly of hexagonal or triangular shape. Intercalation can be performed efficiently and without large-scale destruction of graphene in the studied temperature range between 670 and $870 \mathrm{~K}$. The intercalation of metals was found to occur either via diffusion through preexisting lattice defects in graphene (i.e., domain boundaries, dislocations, and point defects) or through metal-generated defects followed by a subsequent healing process of the graphene lattice. Our findings suggest the possibility of large-scale preparation of graphene-capped and sharply confined ferromagnetic nanoislands with potential technological implications in the fields of spintronics and magnetic data storage.

\section{METHODS}

All STM experiments were carried out in an ultrahigh vacuum (UHV) system (base pressure $1 \times 10^{-10} \mathrm{mbar}$ ) equipped with an Omicron variable-temperature scanning tunneling microscope. All STM measurements were performed in the constant current mode at room temperature using electrochemically etched polycrystalline tungsten tips cleaned in UHV by flash annealing. The sign of the bias voltage corresponds to the voltage applied to the sample. Tunneling current and voltage are labeled $I_{\mathrm{T}}$ and $U_{T}$, respectively. SPIP software was used for STM image processing. The Rh(111) single crystal (MaTeck GmbH, purity 99.99\%) was cleaned by repeated cycles of $\mathrm{Ar}^{+}$sputtering at room temperature $\left(p_{\mathrm{Ar}}=5 \times 10^{-6} \mathrm{mbar}, 1.2 \mathrm{keV}\right)$, flash annealing to about $1500 \mathrm{~K}$, annealing in an oxygen atmosphere $\left(p_{\mathrm{O}_{2}}=1 \times\right.$ $10^{-7} \mathrm{mbar}$ ) at about $1000 \mathrm{~K}$, and subsequent flash annealing in UHV to about $1500 \mathrm{~K}$. The quality of the Rh(111) surface was subsequently checked by STM, LEED, and core-level as well as valence-band PES. Graphene layers were prepared by thermal decomposition of ethene/propene gas $\left(p_{\mathrm{C}_{2} \mathrm{H}_{4} / \mathrm{C}_{3} \mathrm{H}_{6}}=3 \times 10^{-8}\right.$ mbar) at $900-1100 \mathrm{~K}$.

Identical samples were grown for photoemission experiments. The presented valence-band photoemission and corelevel PES measurements were performed at the UE56/2-PGM-1 beamline at BESSY (HZB, Berlin). The PES spectra were collected with a PHOIBOS 100 energy analyzer. The energy/angular resolution was set to $80 \mathrm{meV} / 0.2^{\circ}$.

The intercalation of ferromagnetic material was done in a two-step process. First, Fe and Ni were predeposited from highpurity $\mathrm{Ni}$ or Fe pieces heated by electron bombardment at a deposition rate of about $1 \AA /$ min on graphene/Rh(111) held at room temperature. Second, the samples were annealed between 670 and $870 \mathrm{~K}$ in steps of $10-30 \mathrm{~min}$, leading to the gradual intercalation of material. For the sample shown in Figure 3a, Ni predeposition was performed at $225 \mathrm{~K}$, differing from the other samples, which however has no influence on the further intercalation.
Acknowledgment. This work has been supported by the European Science Foundation (ESF) under the EUROCORES Programme EuroGRAPHENE (Project "SpinGraph"). M.F. gratefully acknowledges the financial support by the Research Center "UltraQuantum" (Excellence Initiative), by the Deutsche Forschungsgemeinschaft (DFG) within the Collaborative Research Center (SFB) 767, and the Baden-Württemberg Stiftung. Y.D. acknowledges the financial support by DFG within research project DE1679/2-1.

\section{REFERENCES AND NOTES}

1. Geim, A. K.; Novoselov, K. S. The Rise Of Graphene. Nat. Mater. 2007, 6, 183-191.

2. Castro Neto, A. H.; Guinea, F.; Peres, N. M. R.; Novoselov, K. S.; Geim, A. K. The Electronic Properties of Graphene. Rev. Mod. Phys. 2009, 81, 109-162.

3. Kim, K. S.; Zhao, Y.; Jang, H.; Lee, S. Y.; Kim, J. M.; Kim, K. S.; Ahn, J.-H.; Kim, P.; Choi, J.-Y.; Hong, B. H. Large-Scale Pattern Growth of Graphene Films for Stretchable Transparent Electrodes. Nature 2009, 457, 706-710.

4. Bae, S.; Kim, H.; Lee, Y.; Xu, X.; Park, J.-S.; Zheng, Y.; Balakrishnan, J.; Lei, T.; Kim, H. R.; Song, Y. I.; et al. Roll-toRoll Production of 30-Inch Graphene Films for Transparent Electrodes. Nat. Nanotechnol. 2010, 5, 574-578.

5. Lee, E. J. H.; Balasubramanian, K.; Weitz, R. T.; Burghard, M.; Kern, K. Contact and Edge Effects in Graphene Devices. Nat. Nanotechnol. 2008, 3, 486-490.

6. Tombros, N.; Jozsa, C.; Popinciuc, M.; Jonkman, H. T.; van Wees, B. J. Electronic Spin Transport and Spin Precession in Single Graphene Layers at Room Temperature. Nature 2007, 448, 571-574.

7. Avsar, A.; Yang, T.-Y.; Bae, S.; Balakrishnan, J.; Volmer, F.; Jaiswal, M.; Yi, Z.; Ali, S. R.; Güntherodt, G.; Hong, B. H.; Beschoten, B.; Özyilmaz, B. Toward Wafer Scale Fabrication of Graphene Based Spin Valve Devices. Nano Lett. 2011, 11, 2363-2368.

8. Karpan, V. M.; Giovannetti, G.; Khomyakov, P. A.; Talanana, M.; Starikov, A. A.; Zwierzycki, M.; van den Brink, J.; Brocks, 
G.; Kelly, P. J. Graphite and Graphene as Perfect Spin Filters. Phys. Rev. Lett. 2007, 99, 176602/1-176602/4.

9. Maassen, J.; Ji, W.; Guo, H. Graphene Spintronics: The Role of Ferromagnetic Electrodes. Nano Lett. 2011, 11, 151-155.

10. Tontegode, A. Ya. Carbon on Transition Metal Surfaces. Prog. Surf. Sci. 1991, 38, 201-429.

11. Shikin, A. M.;Prudnikova, G. V.; Adamchuk, V. K.;Moresco, F.; Rieder, K.-H. Surface Intercalation of Gold Underneath a Graphite Monolayer on Ni(111) Studied by Angle-Resolved Photoemission and High-Resolution Electron-Energy-Loss Spectroscopy. Phys. Rev. B 2000, 62, 13202-13208.

12. Dedkov, Yu. S.; Shikin, A. M.; Adamchuk, V. K.; Molodtsov, S. L.; Laubschat, C.; Bauer, A.; Kaindl, G. Intercalation of Copper Underneath a Monolayer of Graphite on $\mathrm{Ni}(111)$. Phys. Rev. B 2001, 64, 035405/1-035405/6.

13. Riedl, C.; Coletti, C.; Iwasaki, T.; Zakharov, A. A.; Starke, U. Quasi-Free-Standing Epitaxial Graphene on SiC Obtained by Hydrogen Intercalation. Phys. Rev. Lett. 2009, 103, 246804/1-246804/4.

14. Nagashima, A.; Tejima, N.; Oshima, C. Electronic States of the Pristine and Alkali-Metal-Intercalated Monolayer Graphite/Ni(111) Systems. Phys. Rev. B 1994, 50, 17487-17495.

15. Varykhalov, A.; Sánchez-Barriga, J.; Shikin, A. M.; Biswas, C.; Vescovo, E.; Rybkin, A.; Marchenko, D.; Rader, O. Electronic and Magnetic Properties of Quasifreestanding Graphene on Ni. Phys. Rev. Lett. 2008, 101, 157601/1-157601/4.

16. Enderlein, C.; Kim, Y. S.; Bostwick, A.; Rotenberg, E.; Horn, K. The Formation of an Energy Gap in Graphene on Ruthenium by Controlling the Interface. New J. Phys. 2010, 12, 033014/1-033014/9.

17. Sutter, P.; Sadowski, J. T.; Sutter, E. A. Chemistry under Cover: Tuning Metal-Graphene Interaction by Reactive Intercalation. J. Am. Chem. Soc. 2010, 132, 8175-8179.

18. Dedkov, Yu. S.; Fonin, M. Electronic and Magnetic Properties of the Graphene-Ferromagnet Interface. New J. Phys. 2010, 12, 125004/1-125004/22.

19. Varykhalov, A.; Rader, O. Graphene Grown on Co(0001) Films and Islands: Electronic Structure and Its Precise Magnetization Dependence. Phys. Rev. B 2009, 80, 035437/1-035437/6.

20. Eom, D.; Prezzi, D.; Rim, K. T.; Zhou, H.; Lefenfeld, M.; Xiao, S.; Nuckolls, C.; Hybertsen, M. S.; Heinz, T. F.; Flynn, G. W. Structure and Electronic Properties of Graphene Nanoislands on Co(0001). Nano Lett. 2009, 9, 2844-2848.

21. Dedkov, Yu. S.; Fonin, M.; Rüdiger, U.; Laubschat, C. Graphene-Protected Iron Layer on Ni(111). Appl. Phys. Lett. 2008, 93, 022509/1-022509/3.

22. Land, T. A.; Michely, T.; Behm, R. J.; Hemminger, J. C.; Comsa, G. STM Investigation of Single Layer Graphite Structures Produced on $\mathrm{Pt}(111)$ by Hydrocarbon Decomposition. Surf. Sci. 1992, 264, 261-270.

23. Preobrajenski, A. B.; Ng, M. L.; Vinogradov, A. S.; Mårtensson, N. Controlling Graphene Corrugation on Lattice-Mismatched Substrates. Phys. Rev. B 2008, 78, 073401/1-073401/4.

24. Sutter, P.; Sadowski, J. T.; Sutter, E. Graphene on Pt(111): Growth and Substrate Interaction. Phys. Rev. B 2009, 80 , 245411/1-245411/10

25. N'Diaye, A. T.; Bleikamp, S.; Feibelman, P. J.; Michely, T. Two-Dimensional Ir Cluster Lattice on a Graphene Moiré on Ir(111). Phys. Rev. Lett. 2006, 97, 215501/-215501/4.

26. N'Diaye, A. T.; Coraux, J.; Plasa, T. N.; Busse, C.; Michely, T. Structure of Epitaxial Graphene on $\operatorname{Ir}(111)$. New J. Phys. 2008, 10, 043033/1-043033/16.

27. Loginova, E.; Nie, S.; Thürmer, K.; Bartelt, N. C.; McCarty, K. F. Defects of Graphene on $\operatorname{Ir}(111)$ : Rotational Domains and Ridges. Phys. Rev. B 2009, 80, 085430/1-085430/8.

28. Grant, J. T.; Haas, T. W. A Study of Ru(0001) and $\mathrm{Rh}(111)$ Surfaces Using LEED and Auger Electron Spectroscopy. Surf. Sci. 1970, 21, 76-85.

29. Müller, F.; Sachdev, H.; Hüfner, S.; Pollard, A. J.; Perkins, E. W.; Russell, J. C.; Beton, P. H.; Gsell, S.; Fischer, M.; Schreck, M.; et al. How Does Graphene Grow? Easy Access to WellOrdered Graphene Films. Small 2009, 5, 2291-2296.
30. Sicot, M.; Bouvron, S.; Zander, O.; Rüdiger, U.; Dedkov, Yu. S.; Fonin, M. Nucleation and Growth of Nickel Nanoclusters on Graphene Moiré on Rh(111). Appl. Phys. Lett. 2010, 96, 093115/1-093115/3.

31. Wang, B.; Caffio, M.; Bromley, C.; Früchtl, H.; Schaub, R. Coupling Epitaxy, Chemical Bonding, and Work Function at the Local Scale in Transition Metal-Supported Graphene. ACS Nano 2010, 4, 5773-5782.

32. Roth, S.; Orsterwalder, J.; Greber, T. Synthesis of Epitaxial Graphene on Rhodium from 3-Pentanone. Surf. Sci. 2011, 605, L17-L19.

33. Marchini, S.; Günther, S.; Wintterlin, J. Scanning Tunneling Microscopy of Graphene on Ru(0001). Phys. Rev. B 2007, 76, 075429.

34. Vázquez de Parga, A. L.; Calleja, F.; Borca, B.; Passeggi, M. C. G, Jr.; Hinarejos, J. J.; Guinea, F.; Miranda, R. Periodically Rippled Graphene: Growth and Spatially Resolved Electronic Structure. Phys. Rev. Lett. 2008, 100, 056807/1056807/4.

35. Moritz, W.; Wang, B.; Bocquet, M.-L.; Brugger, T.; Greber, T.; Wintterlin, J.; Günther, S. Structure Determination of the Coincidence Phase of Graphene on Ru(0001). Phys. Rev. Lett. 2010, 104, 136102/1-136102/4.

36. Dedkov, Yu. S.; Fonin, M.; Laubschat, C. A Possible Source of Spin-Polarized Electrons: The Inert Graphene/Ni(111) System. Appl. Phys. Lett. 2008, 92, 052506/1-052506/3.

37. Gall, N. R.; Rut'kov, E. V.; Tontegode, A. Ya. Intercalation of Nickel Atoms Under Two-Dimensional Graphene Film on (111)Ir. Carbon 2000, 38, 663-667.

38. Liao, Q.; Zhang, H. J.; Wu, K.; Li, H. Y.; Bao, S. N.; He, P. Nucleation and Growth of Monodispersed Cobalt Nanoclusters on Graphene Moiré on Ru(0001). Nanotechnology 2011, 22, 125303/1-135303/6.

39. Weser, M.; Voloshina, E. N.; Horn, K.; Dedkov, Yu. S. Electronic Structure and Magnetic Properties of the Graphene/Fe/Ni(111) Intercalation-like System. Phys. Chem. Chem. Phys. 2011, 13, 7534-7539.

40. Martoccia, D.; Björck, M.; Schlepütz, C. M.; Brugger, T.; Pauli, S. A.; Patterson, B. D.; Greber, T.; Willmott, P. R. Graphene on $\mathrm{Ru}(0001)$ : A Corrugated and Chiral Structure. New J. Phys. 2010, 12, 043028/1-043028/12.

41. Miniussi, E.; Pozzo, M.; Baraldi, A.; Vesselli, E.; Zhan, R. R.; Comelli, G.; Mentes, T. O.; Niño, M. A.; Locatelli, A.; Lizzit, S.; et al. Thermal Stability of Corrugated Epitaxial Graphene Grown on $\operatorname{Re}(0001)$. Phys. Rev. Lett. 2011, 106, 216101/1216101/4.

42. Brugger, T.; Günther, S.; Wang, B.; Dil, J. H.; Bocquet, M.-L.; Osterwalder, J.; Wintterlin, J.; Greber, T. Comparison of Electronic Structure and Template Function of SingleLayer Graphene and a Hexagonal Boron Nitride Nanomesh on Ru(0001). Phys. Rev. B. 2009, 79, 045407/1045407/6.

43. Malola, S.; Häkkinen, H.; Koskinen, P. Structural, Chemical, and Dynamical Trends in Graphene Grain Boundaries. Phys. Rev. B 2010, 81, 165447/1-165447/6.

44. Lahiri, J.; Batzill, M. Graphene Destruction by MetalCarbide Formation: An Approach for Patterning of MetalSupported Graphene. Appl. Phys. Lett. 2010, 97, 023102/1023102/3.

45. Boukhvalov, D. W.; Katsnelson, M. I. Destruction of Graphene by Metal Adatoms. Appl. Phys. Lett. 2009, 95, 023109/1-023109/3.

46. Coraux, J.; N'Diaye, A. T.; Busse, C.; Michely, T. Structural Coherency of Graphene on $\operatorname{Ir}(111)$. Nano Lett. 2008, 8, 565-570.

47. Manai, G.; Radican, K.; Delogu, F.; Shvets, I. V. RoomTemperature Self-Assembly of Equilateral Triangular Clusters via Friedel Oscillations. Phys. Rev. Lett. 2008, 101, $165701 / 1-165701 / 4$. 